\title{
Face-specific resting functional connectivity between the fusiform gyrus and posterior superior temporal sulcus
}

\author{
Nicholas B. Turk-Browne ${ }^{1 *}$, Samuel V. Norman-Haignere ${ }^{2}$ and Gregory McCarthy ${ }^{2}$ \\ 1 Department of Psychology, Princeton University, Princeton, NJ, USA \\ 2 Department of Psychology, Yale University, New Haven, CT, USA
}

Edited by:

Jennifer S. Beer, University of Texas at Austin, USA

\section{Reviewed by:}

Alexander Todorov, Princeton

University, USA

K. Suzanne Scherf, Carnegie Mellon

University, USA

\section{*Correspondence:}

Nicholas B. Turk-Browne, Department of Psychology, Green Hall, Princeton University, Princeton, NJ 08540, USA. e-mail: ntb@princeton.edu
Faces activate specific brain regions in $\mathrm{fMRI}$, including the fusiform gyrus (FG) and the posterior superior temporal sulcus (pSTS). The fact that the FG and pSTS are frequently co-activated suggests that they may interact synergistically in a distributed face processing network. Alternatively, the functions implemented by these regions may be encapsulated from each other. It has proven difficult to evaluate these two accounts during visual processing of face stimuli. However, if the FG and pSTS interact during face processing, the substrate for such interactions may be apparent in a correlation of the BOLD timeseries from these two regions during periods of rest when no faces are present. To examine face-specific resting correlations, we developed a new partial functional connectivity approach in which we removed variance from the FG that was shared with other category-selective and control regions. The remaining face-specific FG resting variance was then used to predict resting signals throughout the brain. In two experiments, we observed face-specific resting functional connectivity between FG and pSTS, and importantly, these correlations overlapped precisely with the face-specific pSTS region obtained from independent localizer runs. Additional region-of-interest and pattern analyses confirmed that the FG-pSTS resting correlations were face-specific. These findings support a model in which face processing is distributed among a finite number of connected, but nevertheless face-specialized regions. The discovery of category-specific interactions in the absence of visual input suggests that resting networks may provide a latent foundation for task processing.

Keywords: face processing, high-level vision, functional connectivity, fusiform face area, fMRI, scene processing, inferior temporal cortex, rest

\section{INTRODUCTION}

Faces are one of the most important sources of information in the visual environment. They allow us to identify friend from foe, and to interpret the mental states of others. Given this importance, it is no surprise that the brain has evolved specialized mechanisms for processing faces. In fMRI, for example, faces selectively activate several ventral and lateral occipitotemporal cortical regions, most prominently the fusiform gyrus (FG), and the posterior superior temporal sulcus (pSTS).

Focal regions within the FG respond more strongly to faces than to other stimulus categories, such as words, buildings, and objects (Puce et al., 1995; Kanwisher et al., 1997; McCarthy et al., 1997; also known by the functional label: fusiform face area, or FFA). More specifically, the FG may be involved in the processing of facial identity (Winston et al., 2004; Rotshtein et al., 2005). For example, adaptation in the FG to a morphed face is released only to a new morph that crosses an identity boundary (Rotshtein et al., 2005). Neuropsychological studies of prosopagnosia further implicate the FG in identity recognition (Barton et al., 2002), and subdural stimulation of FG sites results in transient prosopagnosia and visual face hallucinations (Allison et al., 1994; Puce et al., 1999).

The pSTS is also routinely observed in fMRI studies of face processing (Puce et al., 1995, 1996; Kanwisher et al., 1997).Compared to the FG, the functional role(s) of the pSTS and surrounding cortex have been more controversial (Hein and Knight, 2008; Mitchell, 2008). The pSTS has been variably associated with many functions, including the perception of biological motion (Allison et al., 2000), social perception (Saxe, 2006), multisensory integration (Ghazanfar and Schroeder, 2006), and spatial attention (Corbetta et al., 2008). However, here we focus on the role of the pSTS in processing socially relevant facial information, such as emotional expressions and eye gaze (Puce et al., 1998; Narumoto et al., 2001; Pelphrey et al., 2004; Engell and Haxby, 2007). In addition to being activated by such stimuli, adaptation and pattern classification studies have revealed coding of specific gaze directions and expressions in the pSTS (Winston et al., 2004; Calder et al., 2007; Furl et al., 2007; Said et al., 2010).

The fact that faces activate many brain regions has led researchers to suggest that faces are processed in a distributed network (Haxby et al., 2000; Ishai, 2008). In such models, the FG and pSTS are core nodes that, respectively, process invariant (identity) and changeable (expression, gaze) facial features. Going back to the cognitive roots of these theories (Bruce and Young, 1986), the processing of different facial features is described as occurring independently in parallel pathways. This view is supported by dissociations between identity and expression processing (Duchaine et al., 2003; Andrews and Ewbank, 2004; Fox et al., 2008). Moreover, evoked responses from subdural electrodes in both regions occur simultaneously, 
without a temporal offset that would provide evidence of feedback/feedforward interactions (Allison et al., 1999). Finally, face processing models in which the FG and pSTS interact provide a somewhat worse fit than purely feedforward models (Fairhall and Ishai, 2007).

At the same time, the independence of the FG and pSTS has been questioned (Calder and Young, 2005; Vuilleumier and Pourtois, 2007). For example, the pSTS can be sensitive to both identity and expression information (Perrett et al., 1984; Hasselmo et al., 1989; Winston et al., 2004), and attention to expression information enhances the response of the FG (Vuilleumier et al., 2004; Ganel et al., 2005). Moreover, even if the computations underlying identity and expression processing (implemented by the FG and pSTS, respectively) were dissociable, interactions between the FG and pSTS may nevertheless prove essential for binding expressions and identities. Indeed, given the multidimensional role of the pSTS in processing facial expressions, eye gaze, and biological motion, it may serve a broader role in deciphering the intentions of social agents (Saxe et al., 2004; Vander Wyk et al., 2009). Thus, while faces activate several brain regions, the relationship between the FG and pSTS may be the most relevant connection in any larger network that tracks individuals and their intentions.

One of the primary challenges for assessing the interactivity/ independence of the FG and pSTS is that both regions respond to faces in a correlated manner (Puce et al., 1995, 1996; Kanwisher et al., 1997). Are such correlations spuriously linked to external face stimulation, or do they reflect internal dynamics within a distributed face processing network? To address this question, we tested the extent to which correlations between the FG and pSTS persist at rest in the absence of external stimulation. Indeed, resting connectivity has been used extensively to characterize cortical networks, based partly on the fact that regions that tend to be activated in unison during tasks also fluctuate in unison at rest (see Corbetta et al., 2008).

In two experiments we examined resting connectivity between the FG and pSTS. Using an independent localizer scan, we identified a right FG seed in each individual that responded selectively to faces, and an adjacent control seed in right ventral visual cortex that responded selectively to images of a different visual category (flowers or scenes in Experiment 1, scenes in Experiment 2). Using separate resting runs, we extracted the BOLD timeseries from the FG and the functional control seed, as well as from several locations in white matter and ventricles whose signal likely reflects non-functional noise and artifacts. The extracted timeseries were entered as regressors into simultaneous multiple regression models of the same resting runs. The resulting parameter estimates for the FG regressor provide a measure of partial functional connectivity, i.e. the degree to which the unique variance in the FG (with respect to the control region and other nuisance regressors) was reflected in other brain regions. Thus, while the resting "task" had nothing in particular to do with faces, we were able to isolate intrinsic activity that was specific to a brain region reliably activated by face stimuli. This approach allows us to observe resting connectivity throughout the whole brain, but we focus on the FG and pSTS in order to characterize the nature of this relationship in detail.
Most previous studies of face-related functional connectivity have examined changes in connectivity across stimulus conditions (Fairhall and Ishai, 2007; Rotshtein et al., 2007; Nummenmaa et al., 2010), rather than baseline intrinsic connectivity that persists at rest. The one study to directly examine face-related resting connectivity relied entirely on exploratory whole-brain analyses (Zhang et al., 2009), without expressly characterizing the specificity and functional significance of the FG-pSTS resting relationship. In particular, they used a control region located in the left FG that is broadly tuned to a range of non-face stimuli and, by being located in the other hemisphere, does not account for hemispheric-specific properties of ventral temporal cortex (e.g., global/local preferences; Fink et al., 1996). Here we use partial connectivity and a combination of whole-brain, region-of-interest, and novel pattern analysis techniques to directly address whether the FG and pSTS share resting variance related to face processing.

These techniques suggest new ways to link task and resting paradigms. Whereas typical single-seed methods have included nuisance regressors for movement and other global factors (see Fox and Raichle, 2007), our study provides an initial demonstration of how regressing out functional regions can reveal more selective connectivity. This partial connectivity approach may be analogous to the canonical subtraction method for assessing the specificity of task-evoked activation. In addition, the pattern analysis technique introduced in Experiment 2 uses resting connectivity to predict the response of brain regions during tasks. This allows us to explicitly test the link between task and resting activations on a region-byregion basis, i.e. by testing whether the task activation in a region can be predicted by the pattern of connectivity in that region to a distinct seed region. The ability to characterize fine-grained networks (cf. gross sensorimotor networks; e.g., Biswal et al., 1995; Lowe et al., 1998) using partial connectivity - and to link this connectivity to task activation - may move us closer to understanding the mechanisms supporting perception and cognition, even in the absence of explicit tasks.

\section{EXPERIMENT 1 MATERIALS AND METHODS Subjects}

Resting and localizer scans were obtained from two existing data sets. Resting scans were only collected in the corresponding studies if time allowed, and so only a subset of the subjects were eligible for the present analysis. We further restricted analysis to subjects for whom we could localize both a right FG seed and right control seed (we failed to localize the FG seed in three subjects and the control seed in two subjects). A total of 12 right-handed subjects with normal or corrected-to-normal vision met these criteria. They received monetary compensation for participating. Informed consent was obtained from all subjects, and the study protocols were approved by the Duke University Medical Center IRB.

\section{Data acquisition and preprocessing}

All data were collected at the Duke Brain Imaging and Analysis Center using a 3T Excite platform GE scanner with an eight-channel head coil. Functional data were collected using a T2*-weighted gradient echo-planar imaging (EPI) sequence with a $2000 \mathrm{~ms}$ repetition time (TR), 30-ms echo time (TE), $60^{\circ}$ flip angle, and a $64 \times 64$ 
matrix. In the first data set, $343.8-\mathrm{mm}$ slices were obtained with a $256 \mathrm{~mm}$ FOV $(4 \times 4 \times 3.8-\mathrm{mm}$ voxels $)$, and in the second data set, $323-\mathrm{mm}$ slices were obtained with a $192-\mathrm{mm}$ FOV $(3 \times 3 \times 3-\mathrm{mm}$ voxels). Two T1-weighted anatomical scans were also collected for spatial registration and normalization (3D and co-planar).

Preprocessing and regression analyses were carried out using FSL 4.1 and FMRIB software libraries (Analysis Group, FMRIB, Oxford, UK). Seeds and regressors were defined using custom scripts for Matlab (Mathworks, Natick, MA, USA). For each localizer and resting scan, the first three volumes were discarded to allow for T1 equilibration. The remaining data were motion-corrected in six dimensions to the middle volume of each run, spatially smoothed using a 6-mm FWHM kernel, and temporally high-pass filtered with a 111-s period. The period of this filter allows frequencies greater than $0.009 \mathrm{~Hz}$, a standard low frequency cutoff across many studies of functional connectivity (e.g., Fox et al., 2005; He et al., 2007; Shulman et al., 2009). We followed this convention, and applied these same preprocessing parameters to both resting and localizer runs. Functional runs were registered to the co-planar structural scan with three degrees of freedom (DOF), which was in turn registered to the high-resolution structural scan with six DOF. Each subject's high-resolution scan was normalized to the MNI template with 12 DOF. Using these transformations, EPI data were transformed into standard space, and interpolated to 2-mm isotropic voxels.

\section{Localizer analysis}

The six subjects in the first data set each completed three localizer runs with 186 volumes at the start of the session. Each localizer run consisted of a pseudo-randomized block design in which 16-s stimulus blocks were interleaved with 16-s blocks of fixation. A total of nine stimulus and fixation blocks were presented in every run, with three each for faces, flowers, and bodies. Stimulus blocks consisted of 16 images from a single category, and subjects were required only to watch the stimuli. The six subjects in the second data set each completed two localizer runs with 196 volumes near the end of the session. Each localizer run consisted of an alternating block design in which 24-s stimulus blocks were interleaved with 6-s blocks of fixation. A total of 12 stimulus and fixation blocks were presented in each run, including six each for faces and scenes. Stimulus blocks consisted of 12 colorful images from a single category, and subjects were required to make a male/female or an indoor/outdoor judgment to each image.

At the first level, each localizer run was fit using a general linear model (GLM) including boxcar regressors convolved with a double-gamma hemodynamic response function for face blocks and control blocks (flowers in the first data set, and scenes in the second data set). Using this GLM, $z$-scores were computed for the contrasts "face $>$ flower" and "flower $>$ face" in the first data set, and "face $>$ scene" and "scene $>$ face" in the second data set. At the second level, statistical maps were combined across runs of a given subject using a fixed-effects model.

Two seeds were defined in each subject using the second-level contrast maps. Seeds were defined separately for each subject due to the anatomical variability of the corresponding regions in past studies. This general approach is a highly conventional way to define ROIs in the ventral visual stream. First, a right FG seed was defined as the peak voxel in a cluster centered on the right mid-fusiform gyrus from the "face $>$ flower" and "face $>$ scene" contrasts. Second, a control seed was defined in each subject as the peak voxel in a cluster adjacent to the FG seed in the right hemisphere from the "flower > face" and "scene > face" contrasts. Flowers have been used in previous studies as a control object for face processing, and regions activated by flowers are generally responsive to most non-face objects (McCarthy et al., 1997). Scenes have been used extensively to define a category-selective region of parahippocampal cortex (PHC) in the collateral sulcus (Aguirre et al., 1998; Epstein and Kanwisher, 1998; also known by the functional label: parahippocampal place area, or PPA).

While the "control" categories for the two data sets were different, flower- and scene-selective regions were proximal to each other and to the FG. Most importantly, the flower and scene seeds were never combined in any way: a flower seed was used as the control region for subjects in the first data set, and a scene seed was used as the control region for subjects in the second data set. We hypothesized that either region would control for generic aspects of visual processing. Moreover, we believed that our conclusions would be strengthened by showing that any patterns of FG resting connectivity did not depend on our particular choice of control region. In Experiment 2, we replicate the study in a homogenous sample of subjects using only the scene control region (i.e. PHC).

The reliability of the localizer results across subjects was assessed at the third level by combining second-level maps using FSL's standard approach for random effects analyses (FLAME1). FLAME is a more sophisticated version of the standard ordinary least square GLM in which the random effects component of the mixed effects model is estimated using Bayesian techniques (Woolrich et al., 2004). To correct for multiple comparisons, the two-stage cluster correction in FSL was used: first, an initial $z$-score threshold $(z>1.65)$ was applied to every voxel in order to determine which clusters entered the second stage, and then cluster significance was determined by a Gaussian random fields method based on the smoothness of the data (Poline et al., 1997; Woolrich et al., 2004). The resulting group statistical maps were cluster-thresholded to a corrected alpha of $p<0.05$. We only computed the third level statistical map for the second level contrasts "face $>$ flower" and "face $>$ scene" because we expected to obtain compatible face selective regions in both contrasts (while the reverse was not necessarily true: flower and scene regions need not overlap). Specifically, this third level group map was used to compare the locations of the face-selective pSTS region from the localizer with any $\mathrm{pSTS}$ region obtained from the resting functional connectivity analysis described below.

\section{Resting voxelwise analysis}

In the first data set, five subjects completed two resting runs, and one subject completed one resting run. For all but two subjects, 180 volumes were acquired in each resting run (and 196/212 volumes for the remaining two subjects). In the second data set, two subjects completed two resting runs, and four subjects completed one resting run. All resting runs in this study contained 186 volumes. Thus, in total, seven subjects completed two resting runs and five subjects completed one resting run. These runs were collected at the end of each scanning session, and subjects were instructed to fixate on a central point. 
To analyze resting functional connectivity, the FG and control seeds were overlaid on the preprocessed resting data (see Figure 1). We extracted the time course of the BOLD signal from these seeds by computing a weighted-mean for each volume across voxels, with weights determined by an 8-mm FWHM Gaussian kernel centered on each seed. The resulting timeseries were then entered as regressors in a new voxelwise GLM of each resting run, with the goal of identifying voxels that share variance with the FG.

Because the FG and control regressors (and the other nuisance regressors described below) were entered simultaneously in the GLM, only variance unique to each regressor contributed to its beta (or parameter estimate). In other words, as in standard regression, the parameter estimate associated with each regressor reflects the extent to which the unique variance in that regressor explains the variance remaining in each voxel after fitting all other regressors (Meyers et al., 2006). Essentially, this corresponds to a separate stepwise regression for each regressor in which it is entered last into the model. To further validate our approach, we explicitly orthogonalized the FG regressor with respect to other regressors before conducting the analysis and obtained identical results. Thus, the parameter estimate for the FG regressor in a particular voxel reflected the partial correlation between the resting time course of that voxel and the FG regressor, independent of the other regressors. By including a functionally defined control seed from right ventral visual cortex as a regressor, we partialed out variance in the FG related to generic visual processing and high-level object processing. We thus operationally define the residual unique FG variance as face-specific.

To further partial out FG variance reflecting global sources of noise, we included several other nuisance regressors that are often used in whole-brain connectivity analyses (Fox et al., 2005). These include: the global mean BOLD time course over all voxels, the six movement parameters from motion correction, the BOLD time course from four white matter seeds (bilateral anterior and posterior), the BOLD time course from four ventricle seeds (bilateral anterior and posterior), and the temporal derivatives of all nuisance regressors. Thus, each resting run was modeled at the first-level by 32 regressors (see Figure A1 in Appendix for an example resting run design matrix, and Figure A2 in Appendix for a group confusion matrix of all regressors). In addition, all resting data and regressors were bandpass filtered from .009 to $.08 \mathrm{~Hz}$ (e.g., Fox et al., 2005).

The first level GLM was used to derive $z$-scores for the fit between each voxel and the FG regressor. At the second level, statistical maps corresponding to the partial functional connectivity with the FG were then combined across all runs for a given subject using a fixed-effects approach. The reliability of these second-level maps was assessed across subjects at the third level using a mixed-effects approach (FLAME1), and the resulting group statistical maps were cluster-thresholded ( $p<0.05$, corrected).

\section{RESULTS AND DISCUSSION}

The group analysis of the resting runs revealed significant FG partial functional connectivity in the right pSTS (Figure 2A). Importantly, this cluster found during rest overlapped considerably with a face-selective cluster obtained from the independent

\section{${ }^{\text {A }}$ Localizer Model}
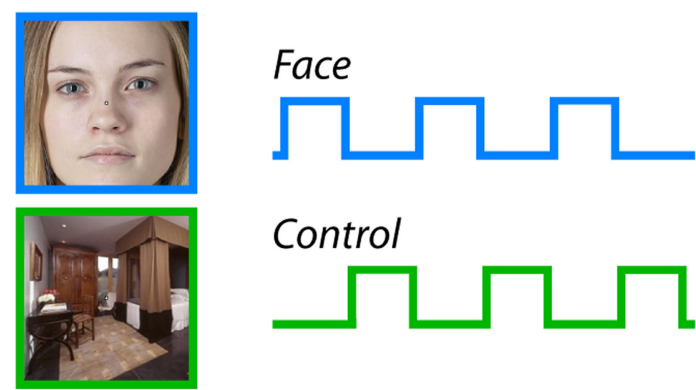

\section{Control}

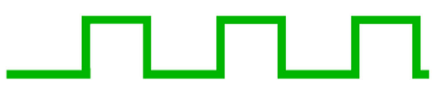

\section{Bxample Subject Seeds}

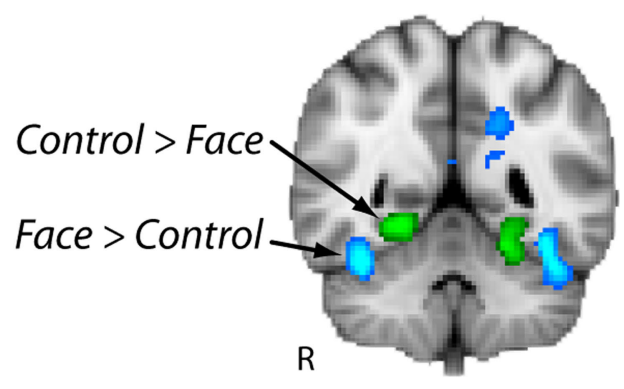

${ }^{c}$ Resting Model

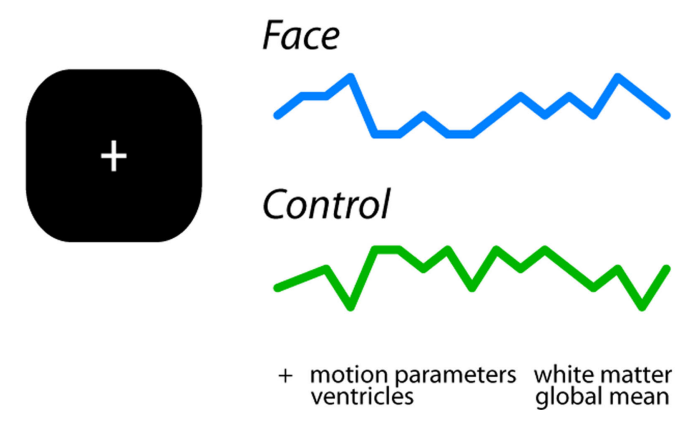

FIGURE 1 |Analysis pipeline. (A) Localizer runs were modeled with regressors for face blocks and control blocks (scenes or flowers in Experiment 1, scenes in Experiment 2). (B) The contrast of these two regressors identified two seed regions in each subject for further investigation: the right fusiform gyrus (FG), and an adjacent region of right ventral temporal cortex selective for flowers or scenes (the latter is the PHC). (C) Timeseries were extracted from these regions in each resting run, and entered simultaneously in a GLM of the same run (along with other nuisance regressors of no interest).

localizer runs (Figure 2B). Given the broad spatial variability of face-specific pSTS regions in similar analyses of evoked responses (Allison et al., 2000; Hein and Knight, 2008), our results provide compelling evidence for coupling of task and resting networks.

Given our a priori interest in the FG and pSTS we focused our analyses on these two regions. However several other clusters of correlated activity were observed, including: left FG, lateral occipital 


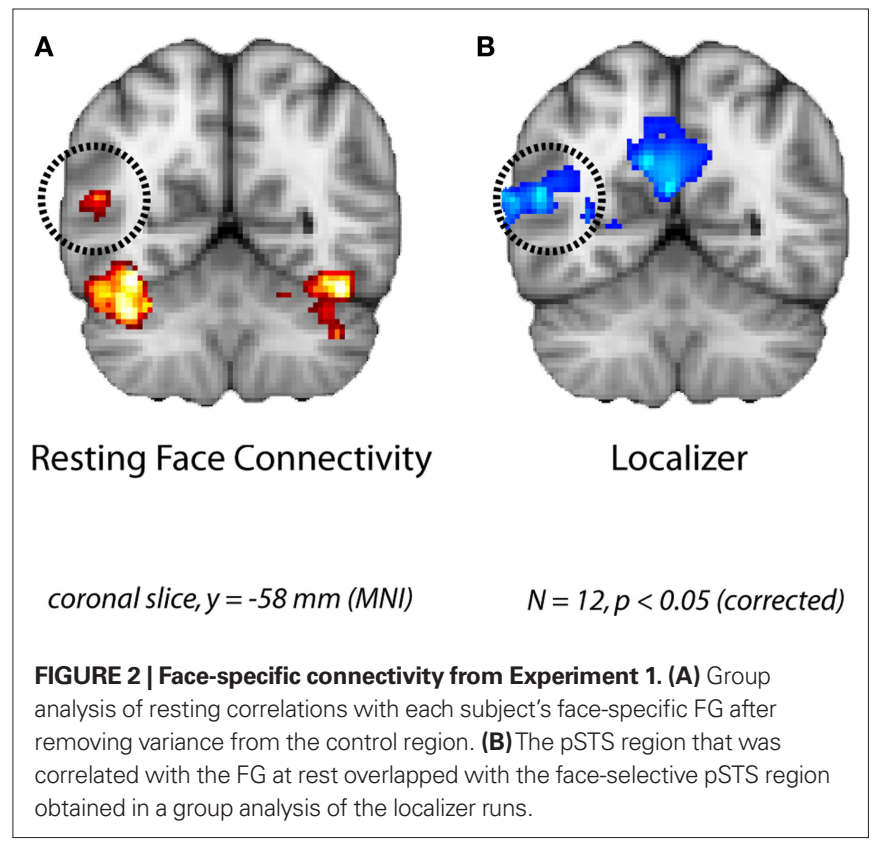

cortex, inferior occipital gyrus, dorsal and ventral striatum, thalamus, bilateral hippocampus, and left amygdala (see Table $\mathbf{1}$ for coordinates). The left FG in particular serves as a sanity check due to its shared face-specific function with the right FG, and its relative anatomical distance from the right FG. In addition, clusters in orbitofrontal cortex and frontal pole were anti-correlated with the right FG seed.

\section{EXPERIMENT 2 \\ MATERIALS AND METHODS \\ Subjects}

To replicate and extend our findings of Experiment 1, we collected resting and localizer data from a new group of 18 naive subjects. All subjects were right-handed, had normal or corrected-to-normal vision, and received monetary compensation for participating. Informed consent was obtained from subjects, and the study protocol was approved by the Human Investigation Committee of the Yale School of Medicine.

\section{DATA ACQUISITION AND PREPROCESSING}

All data were collected at the Yale Anlyan Center using a 3T Siemens Trio scanner with an eight-channel head coil. Functional data was collected using a T2* -weighted gradient EPI sequence with a $1500 \mathrm{~ms}$ TR, $25 \mathrm{~ms} \mathrm{TE}, 90^{\circ} \mathrm{flip}$ angle, and a $64 \times 64$ matrix. In a given volume, 26 5 -mm slices parallel to the anterior commissure-posterior commissure line were obtained with a $224-\mathrm{mm}$ FOV $(3.5 \times 3.5 \times 5 \mathrm{~mm}$ voxels $)$. Two T1-weighted anatomical scans were also collected for spatial registration and normalization (3D and co-planar). Exploratory spectral analyses of Experiment 1 (not reported here) revealed that relatively high-frequency oscillations above $0.05 \mathrm{~Hz}$ contained meaningful signal, and thus temporal filtering was not used in Experiment 2. Our use of partial correlations eliminates concerns about high-frequency artifacts since it controls for shared variance across all frequencies. Otherwise, all preprocessing parameters and routines were identical to Experiment 1.
Table 1 | Resting partial FG connectivity in Experiment 1.

\begin{tabular}{llrrr}
\hline Region & & \multicolumn{1}{c}{$\mathbf{x}$} & y & \multicolumn{2}{c}{ z } \\
\hline POSITIVE CORRELATIONS & & & & \\
Posterior superior temporal sulcus & $\mathrm{R}$ & 50 & -58 & 14 \\
Fusiform gyrus & $\mathrm{L}$ & -38 & -50 & -20 \\
Inferior occipital gyrus & $\mathrm{R}$ & 44 & -74 & -16 \\
& $\mathrm{~L}$ & -42 & -74 & -18 \\
Lateral occipital cortex & $\mathrm{R}$ & 46 & -76 & 0 \\
& & 42 & -76 & 12 \\
& $\mathrm{~L}$ & -40 & -82 & 0 \\
Hippocampus & & -38 & -68 & 12 \\
& $\mathrm{R}$ & 20 & -28 & -6 \\
Amygdala & $\mathrm{L}$ & -20 & -18 & -16 \\
Anterior cingulate & & -18 & -34 & -6 \\
Putamen & $\mathrm{L}$ & -26 & -4 & -24 \\
Caudate body & & 0 & -4 & 28 \\
Caudate head/nucleus & $\mathrm{R}$ & 32 & 0 & -4 \\
Thalamus & $\mathrm{L}$ & -14 & 0 & 20 \\
Pons & $\mathrm{L}$ & -8 & 10 & 2 \\
Brainstem & $\mathrm{L}$ & -4 & -14 & 4 \\
Cerebellum & & -4 & -24 & -30 \\
NEGATIVE CORRELATIONS & & 2 & -20 & -10 \\
Frontal pole & $\mathrm{L}$ & -16 & -72 & -30 \\
& $\mathrm{R}$ & 28 & 56 & -12 \\
& $\mathrm{~L}$ & -28 & 62 & 2 \\
\hline
\end{tabular}

Peak MNI coordinates from clusters correlated with the FG regressor after removing variance from the control regressor (corrected $p<0.05$ ).

\section{Localizer analysis}

One localizer run containing 294 volumes was collected for each subject. Each localizer run consisted of an alternating block design in which 18-s stimulus blocks were interleaved with 18-s blocks of fixation. A total of 12 stimulus and fixation blocks were presented in each run, including six each for faces and scenes. Stimulus blocks consisted of 12 grayscale images from a single category, and subjects were required to detect back-to-back repetitions of an image. The localizer run was modeled and analyzed in the same way as Experiment 1, with one point worth emphasizing: the control category for every subject was scenes, and thus the PHC served as the sole control seed for the resting functional connectivity analyses described below. The FG and PHC seeds could be defined in every subject.

\section{Resting voxelwise analysis}

All subjects completed a single resting run containing 294 volumes in which they fixated a central point. Resting timeseries from the FG and PHC were extracted in the same manner as in Experiment 1 , and whole-brain functional connectivity was assessed using an isomorphic, but slightly modified two-step multiple regression approach: we first fit data with all nuisance and control regressors and then performed whole-brain and ROI analyses on the residual from this GLM. This change permitted us to extract partial ROI timeseries that were orthogonal to the control region and nuisance variables, to mirror the variance fit in the whole-brain analysis. Due 
to the larger sample size, we used a higher voxel threshold $(z=2.3)$ at the first stage of cluster-correction. The corrected alpha after the second stage was identical $(p<0.05)$.

\section{Resting ROI analysis}

In addition to the whole-brain analyses reported in Experiment 1, we also conducted an ROI analysis to further explore the relationship between the FG and the pSTS. Specifically, we compared the strength of the pSTS's correlation with the FG vs. the PHC. The previous analyses tested whether face-specific FG-pSTS resting connectivity exists, and this analysis tests whether pSTS connectivity is widespread vs. more exclusive to the FG.

For this analysis, we localized the peak face-selective voxel in the FG and the pSTS and the peak scene-selective voxel in the PHC in each subject. While the FG and PHC were found in every subject (as described above), the pSTS could only be localized in $17 / 18$ subjects and thus ROI analyses are reported for only those subjects. Timeseries were extracted from these regions using an 8-mm FWHM Gaussian-weighted average of voxels surrounding the peak, as was done for the whole-brain analysis. To account for the possibility that the selectivity of the resulting signals was weakened by this averaging, parallel analyses were conducted with just the peak voxel from each region. In both cases, a facespecific FG timeseries was produced for each subject by removing the raw PHC (and nuisance) resting timeseries from the raw FG resting timeseries using the regression model from the wholebrain analysis. Analogously, the raw FG (and nuisance) resting timeseries were removed from each subject's raw PHC resting timeseries, resulting in a scene-specific PHC timeseries. These partial timeseries were then separately correlated with the pSTS resting timeseries. A reliable correlation implies that the pSTS shares variance with the FG that is independent of the PHC (and vice versa). The FG-pSTS and PHC-pSTS correlation coefficients were converted to $\mathrm{z}$ scores using Fisher's $r$-to- $z$ transformation. The reliability of the correlations was assessed across subjects using one-sample $t$-tests against a null hypothesis of 0 , and the FG-pSTS and PHC-pSTS correlations were directly compared using a dependent-samples $t$-test.

\section{Resting/task pattern analysis}

While the ROI analysis examined the strength of the pSTS correlation with the FG and PHC, it did not directly address whether FG or PHC correlations with pSTS are related to face processing. We therefore developed a novel pattern analysis approach to further characterize the face-specificity of pSTS connectivity. In particular, across voxels in the pSTS we tested how well the pattern of contrast values from the localizer (face $>$ scene) predicted the pattern of partial correlations with the FG vs. the pattern of partial correlations with the PHC. We hypothesized that if FG-pSTS connectivity is related to face processing, then pSTS voxels that are more selective for faces in the localizer should be more correlated with the FG at rest (a positive correlation between the localizer contrast and the amount of FG connectivity). Moreover, we hypothesized that if PHC-pSTS connectivity is unrelated to face processing, then the face-selectivity of pSTS voxels in the localizer should not predict the strength of correlation with the PHC (a zero correlation). Note that this PHC analysis could also yield a negative correlation in which the scene-selectivity of pSTS voxels in the localizer (negative contrast values) would be correlated with greater PHC connectivity.

The two most intuitive ways to select pSTS voxels that will form the patterns for this analysis would be from the group resting FG connectivity map, or from the group face > scene localizer contrast. Note, however, that neither of these methods is independent of the analysis described above: the parameter estimates used to select the pSTS voxels of interest would also be used to compute the new correlation. Such non-independence could potentially bias the results in favor of our hypothesis. Given that there was a reliable PHC-pSTS partial correlation in the ROI analysis, we were able to use a conservative approach: to form pSTS patterns, we selected a population of pSTS voxels that showed reliable partial correlations with the PHC. Selecting voxels in this way is conservative because the manner in which the voxels were selected is independent of the pattern of FG connectivity. If anything, this analysis could be biased in favor of finding an effect for the PHC rather than the FG because the PHC connectivity pattern was likely to have less noise. Note also that our relatively large voxel sizes and the application of spatial smoothing during preprocessing might reduce the amount of information carried in spatial voxel patterns. Critically, this works against us, and applies equally to the FG and PHC connectivity patterns.

While we knew that the PHC correlated with the peak pSTS voxel, to obtain a bigger population of pSTS voxels we seeded the whole-brain with the partial PHC timeseries. We do not report additional results from this analysis because of the present focus on face processing; we are exploring the existence of a resting scene network in ongoing studies (see also Nir et al., 2006). We selected a cluster of voxels in the pSTS from the group resting connectivity map for the control PHC regressor (604 voxels, $p<0.001$ ). Because the pSTS voxels were selected at the group level, we apply this analysis to all 18 subjects (despite the lack of a face-specific pSTS ROI in one subject). We extracted three values from each pSTS voxel in every subject: the difference in parameter estimates for faces vs. scenes from the localizer (resulting in a localizer contrast map), the resting partial correlation with the FG (resulting in an FG connectivity map), and the resting partial correlation with the PHC (resulting in a PHC connectivity map). Only the single peak FG or PHC voxel was used to compute partial correlations with the pSTS because these seeds were being compared to single pSTS voxels, and because the previous analysis revealed that selectivity was partially diluted by the kernel. We compared the resulting pSTS voxel patterns by correlating: (1) the localizer contrast map with the FG connectivity map, (2) the localizer contrast map with the PHC connectivity map, and (3) the localizer contrast map with the difference map of FG-PHC connectivity. The resulting correlation coefficients were converted to $z$ scores, and compared across subjects with $t$-tests.

\section{RESULTS AND DISCUSSION}

In an effort to replicate the finding of FG-pSTS resting connectivity in Experiment 1, we conducted a second focused experiment with a new sample of subjects. We repeated the same whole-brain analysis, this time using a single control region (the scene-selective PHC). Again, we observed a right pSTS region that showed par- 
tial functional connectivity with the FG (Figure 3A). The peak of this region was slightly anterior to the peak of Experiment 1, but importantly, it overlapped with the face-selective pSTS region from the independent localizer runs in Experiment 2 (Figure 3B). Moreover, right FG was again reliably correlated with left FG (see Table 2 for additional regions and coordinates). These results further support the existence of a latent face-specific network including FG and pSTS.

To quantify the spatial consistency of the pSTS obtained from resting and localizer analyses, we compared the location of the group resting connectivity pSTS to the locations of pSTS ROIs defined in each subject from the localizer (to be used in the ROI analysis below). Subtracting the resting connectivity peak from the mean location of the localizer ROIs resulted in differences of -3.90 , $-6.45,7.41 \mathrm{~mm}$, along the $x, y$, and $z$ dimensions respectively. We further evaluated the distance of the resting connectivity peak from the 3-D cluster of pSTS ROIs. We first computed the Mahalanobis distance, taking into account the variance and covariance among spatial dimensions to produce a normalized squared distance from the cluster center (Mahalanobis distance $=2.98$ ). In order to determine whether the resting connectivity peak was a multivariate outlier from the ROI cluster, we statistically evaluated this distance as Chi-squared with degrees of freedom equal to the number of dimensions $\left(\chi^{2}[3]=2.98, p=0.16\right)$. Based on the typical critical value for Mahalanobis distance outliers of $p<0.001$ (Tabachnick and Fidell, 2007), we cannot reject the null hypothesis that the resting connectivity pSTS peak is part of the same spatial distribution as the localizer ROI pSTS peaks.

By using a single well-characterized control region, we were also able to explore how the face-selective pSTS correlated with a region selective for a different visual category. To mirror the initial whole-brain analyses, we used a Gaussian kernel to generate the timeseries for the FG, PHC, and pSTS defined from the localizer. We observed a reliable FG-pSTS correlation across subjects (mean $z=0.15, t[16]=2.85, p=0.011$ ). Interestingly,

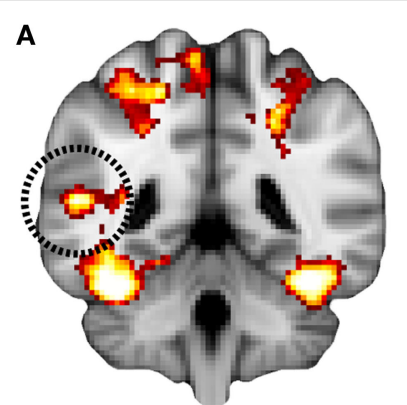

Resting Face Connectivity

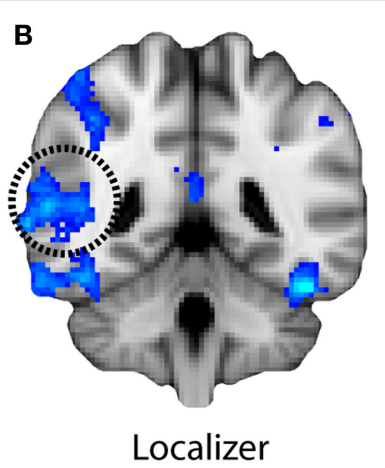

$N=18, p<0.05$ (corrected)
Table 2 | Resting partial FG connectivity in Experiment 2.

\begin{tabular}{|c|c|c|c|c|}
\hline Region & & $\mathbf{x}$ & y & $\mathbf{z}$ \\
\hline \multicolumn{5}{|l|}{ POSITIVE CORRELATIONS } \\
\hline Posterior superior temporal sulcus & $\mathrm{R}$ & 54 & -44 & 10 \\
\hline Fusiform gyrus & $\mathrm{L}$ & -30 & -52 & -22 \\
\hline Inferior occipital gyrus & $\mathrm{R}$ & 36 & -76 & -14 \\
\hline Middle superior temporal sulcus & $\mathrm{R}$ & 48 & -20 & -10 \\
\hline \multirow[t]{2}{*}{ Lateral occipital cortex } & $\mathrm{R}$ & 50 & -68 & -6 \\
\hline & $\mathrm{L}$ & -38 & -78 & -4 \\
\hline Medial temporal lobe & $\mathrm{R}$ & 36 & -6 & -30 \\
\hline \multirow[t]{2}{*}{ Transverse occipital sulcus } & $\mathrm{R}$ & 30 & -72 & 20 \\
\hline & $L$ & -20 & -80 & 22 \\
\hline \multirow[t]{2}{*}{ Superior parietal lobule } & $\mathrm{R}$ & 28 & -48 & 54 \\
\hline & $\mathrm{L}$ & -28 & -48 & 52 \\
\hline Precentral gyrus & $\mathrm{R}$ & 54 & 4 & 34 \\
\hline \multicolumn{5}{|l|}{ NEGATIVE CORRELATIONS } \\
\hline Medial frontal gyrus & $\mathrm{R}$ & 4 & 32 & 36 \\
\hline \multirow[t]{2}{*}{ Angular gyrus } & $\mathrm{R}$ & 64 & -46 & 40 \\
\hline & $\mathrm{L}$ & -58 & -48 & 42 \\
\hline \multirow[t]{2}{*}{ Orbital frontal cortex } & $\mathrm{R}$ & 42 & 26 & -10 \\
\hline & $L$ & -46 & 34 & -8 \\
\hline Inferior frontal gyrus & $\mathrm{R}$ & 48 & 18 & 6 \\
\hline Middle frontal gyrus & $\mathrm{R}$ & 48 & 22 & 44 \\
\hline \multirow[t]{2}{*}{ Superior frontal gyrus } & $\mathrm{R}$ & 22 & 16 & 60 \\
\hline & $L$ & -24 & 14 & 60 \\
\hline \multirow[t]{2}{*}{ Anterior insula } & $\mathrm{R}$ & 36 & 20 & -4 \\
\hline & $\mathrm{L}$ & -32 & 16 & -8 \\
\hline
\end{tabular}

Peak MNI coordinates from clusters correlated with the FG regressor after removing variance from the control regressor (corrected $p<0.05$ ).

the correlation between the PHC and pSTS also reached significance (mean $z=0.064, t[16]=2.58, p=0.020$ ). The directional hypothesis that the FG shares more variance with the pSTS than the PHC approached significance $(t[16]=1.52$, one-tailed $p=0.075)$. Since the spatial smoothing of the Gaussian kernels (blind to the underlying functional data) may have diluted the selectivity of the peak localizer voxels, we performed a parallel ROI analysis over just the peak voxels. The results for the two approaches agreed, although as hypothesized they were more robust at the peak: FG-pSTS correlation (mean $z=0.18$, $t[16]=2.95, p=0.0094)$, PHC-pSTS correlation (mean $z=0.063$, $t[16]=2.21, p=0.042)$; the paired comparison reached significance $(t[16]=1.77$, one-tailed $p=0.048)$. These results are shown in Figure 4. For the sake of completeness, we also verified that the whole-brain results held without using a kernel (Figure A3 in Appendix).

The FG-pSTS connectivity replicated the whole-brain results using individual subjects' pSTS ROIs and without depending on spatial overlap across subjects; this suggests that our primary results are robust across analysis approaches. The PHC-pSTS connectivity was somewhat unexpected, but note that the FG and $\mathrm{PHC}$ timeseries were orthogonalized with respect to each other, and therefore the correlations reflect independent sources of variance within the pSTS. Finally, while the whole-brain results 


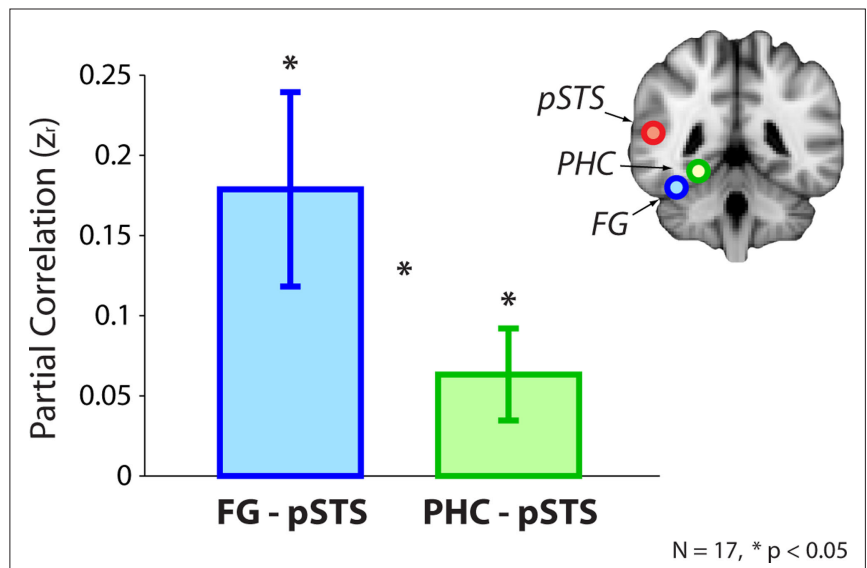

FIGURE 4 | ROI analysis from Experiment 2. Resting correlations between the FG (after partialing out the PHC) and the pSTS, and the PHC (after partialing out the FG) and the pSTS. Correlations were computed withinsubject using peak voxels from the localizer and converted to z-scores for statistical tests. Error bars reflect standard errors of the mean.

support the novel conclusion that there exists variance in the FG that is unique with respect to the $\mathrm{PHC}$ and correlates with the pSTS, the ROI results not only support this conclusion, but provide preliminary support for the additional conclusion that there exists more variance in the (face-specific) pSTS related to the FG than to the PHC. Nevertheless, the paired comparison between the FG and PHC was weakly significant, and so we explored more incisive ways to test the face-selectivity of FG-pSTS resting interactions.

The fact that the reliable FG-pSTS and PHC-pSTS correlations reflected statistically independent sources of variance suggests that they may represent functionally distinct relationships. In particular, we hypothesized that only FG-pSTS connectivity would be related to face processing in the pSTS region. To test this prediction, we developed a novel pattern analysis to examine the relationship between localizer activation and resting connectivity within the pSTS. Using the localizer contrast (face $>$ scene) as a measure of face-selectivity, we hypothesized that the pattern of localizer effects across pSTS voxels should be correlated with the pattern of correlations with the FG across pSTS voxels, but that the same should not be true for the pattern of correlations with the PHC across pSTS voxels. Indeed, across a population of pSTS voxels - selected using an independent and conservative approach (see section Methods) - the strength of the localizer contrast (face $>$ scene) in a given pSTS voxel was positively correlated with the strength of connectivity between that voxel and the peak FG (mean $z=0.15, t[17]=3.98$, $p<0.001)$. This relationship did not hold between a voxel's localizer effect and connectivity with the peak PHC (mean $z=0.0064, t[17]=0.094, p=0.93)$. The directional hypothesis that the localizer pattern should better predict the FG vs. PHC connectivity pattern reached significance $(t[17]=1.89$, one-tailed $p=0.038$ ), as did an analysis in which the localizer pattern (face - scene) was correlated with a connectivity difference pattern (FG-PHC connectivity; $t[17]=2.15$, one-tailed $p=0.023)$. These results are shown in Figure 5 .
Note that the direction of the localizer effect entered into these analyses (face > scene) only impacts the direction of the resulting correlation. Thus the null effect for the PHC suggests no relationship between either face- or scene-selectivity in the pSTS and connectivity with the PHC. These results provide strong evidence that FG-pSTS resting correlations are related to face processing.

\section{GENERAL DISCUSSION SUMMARY}

The goal of our study was to explore the existence of face-specific resting functional connectivity between the FG and pSTS. Across two experiments, we observed resting correlations between these two regions after controlling for many confounding factors such as the visual responsiveness of both regions and generic sources of whole-brain noise. Analogous to how category-specific visual processing is typically studied, we tested whether these correlations were face-specific by using another visual category as a control. In particular, we removed variance from the FG that was shared with a region specialized for the control category before computing the correlations with the pSTS. Additional ROI and pattern analyses further supported our interpretation of the FG-pSTS correlations as face-specific. In the remainder of this section, we discuss issues related to this interpretation, and then consider our findings in the broader context of the face processing and resting state literatures.

\section{EVIDENCE FOR FACE-SPECIFICITY}

We interpreted the observed FG-pSTS resting connectivity as facespecific because it was obtained after removing the resting activity from a single category-specific control region, e.g., the sceneselective PHC. In principle, however, activity related to the processing of other categories - bodies, cars, word-strings, etc. - may have remained in the FG, thus affecting our claim that FG connectivity with the pSTS can be face-specific. We do not intend to claim that the observed FG-pSTS resting connectivity was $100 \%$ face-specific as a result of using one control region. However, several features of our approach and results help establish this specificity.

Most apparent to us is that this criticism could be leveled against almost every study involving category-specific ROIs: how can one be certain that a control condition contains all features of a stimulus not specific to the category of interest - why stop with bodies and cars, what about hair, animal faces, round objects, living things, etc.? One answer is that, in practice, scenes are an especially good control for face processing, and have been used extensively for this reason in functional localizers of the face-specific FG (e.g., Cox et al., 2004; Yi et al., 2004; Gazzaley et al., 2005; Turk-Browne et al., 2006). Moreover, in some ways our approach is more conservative than claiming face-specificity from a localizer contrast: despite showing greater responses to faces than scenes, the FG still shows some response above baseline to scenes (Johnson et al., 2007); orthogonalizing the response of the FG with respect to the PHC removes this variance. Finally, variance related to other categories could only influence our results if it was present in the FG and pSTS, but not the PHC - otherwise it would be removed by our partial correlation procedure.

In addition to these facts about the literature and our design, several results from our study support the conclusion that some component of FG-pSTS resting connectivity is related to face 


\section{pSTS Voxel Patterns}

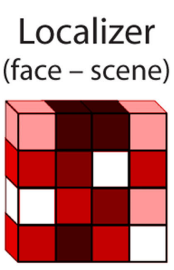

Localizer

FG Connectivity

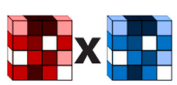

FG Connectivity

(correlation w/ peak FG)

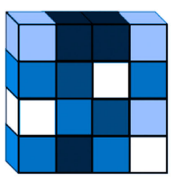

Localizer

PHC Connectivity

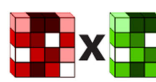

PHC Connectivity

(correlation w/ peak PHC)

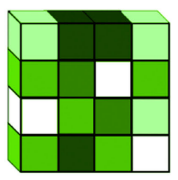

Localizer
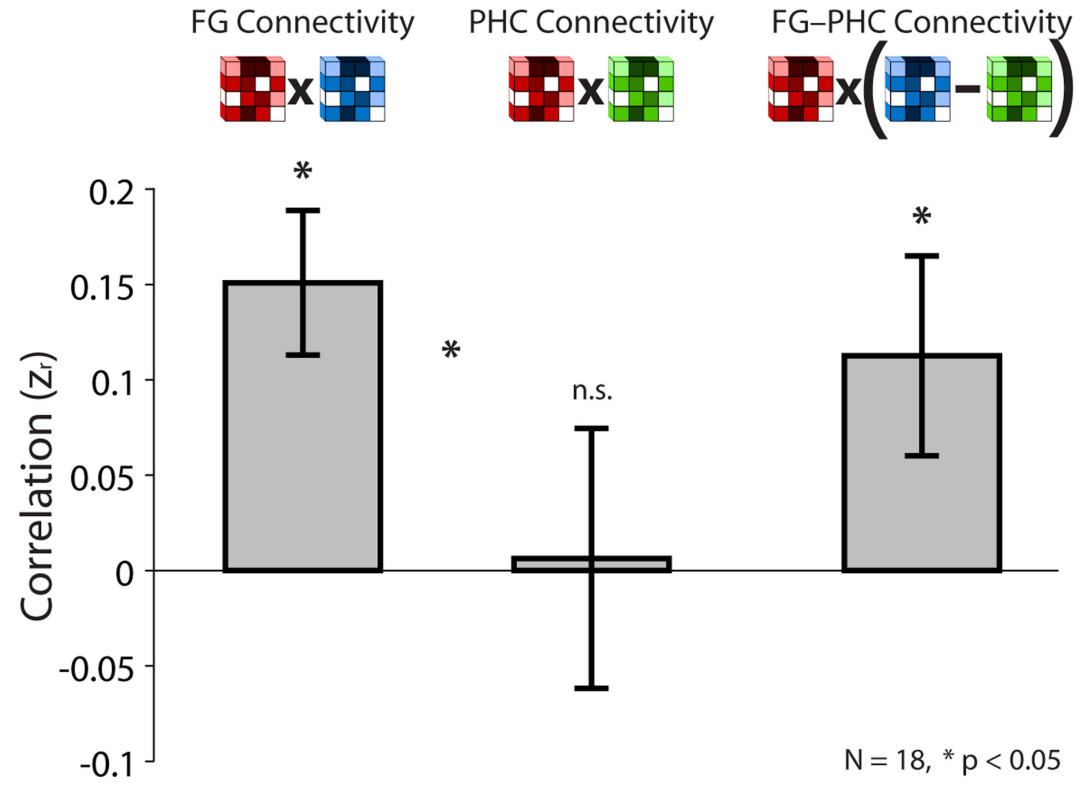

FIGURE 5 | Pattern analysis from Experiment 2. Across a population of pSTS voxels, correlations between the strength of face-selectivity from the localizer and the strength of resting connectivity with the peak FG (after partialing out the $\mathrm{PHC}$ ) and the peak PHC (after partialing out the FG). Voxels that were more face-selective in the localizer were also more correlated with the FG at rest, but there was no such relationship in either direction for the PHC. Moreover, face-selectivity in the localizer (face-scene) predicted face-selectivity in partial resting connectivity (FG-PHC connectivity). Correlations were computed within-subject and converted to $z$-scores for statistical tests. Error bars reflect standard errors of the mean. processing. First, the correlated pSTS region in both experiments overlapped with the pSTS region that showed face-selective evoked responses in the independent localizer scans of the same subjects. Second, beyond demonstrating the there exists some FG-pSTS partial correlation, the weaker PHC-pSTS correlation in Experiment 2 suggests that the relationship with the FG is special. Third, despite qualitative differences in the measures obtained from the resting scans (correlations in spontaneous fluctuations from the FG) and the localizer scans (evoked responses to face vs. house images), the pattern of resting connectivity with the FG in the pSTS - but not with the PHC - was predictive of the pattern of face-selectivity from the localizer in the pSTS. These latter results provide especially compelling evidence that the resting FG-pSTS relationship may have functional significance for face processing.

\section{FACE PROCESSING}

Faces convey multiple types of information, including: identity, gaze direction, emotional expression, attractiveness, biological motion, and speech. Processing of these distinct features has been mapped onto different brain regions, which have in turn been organized hierarchically in distributed models of face processing (Haxby et al., 2000; Ishai, 2008). While there has been disagreement over the degree of face-specificity and modularity of regions in the face network (Hanson et al., 2004; Wiggett and Downing, 2008), perhaps the two most reliable nodes across studies are the FG (Puce et al., 1995; Kanwisher et al., 1997; McCarthy et al., 1997), involved in processing identity (Allison et al., 1994; Barton et al., 2002; Rotshtein et al., 2005), and the pSTS (Puce et al., 1995, 1996; Kanwisher et al., 1997), involved in processing eye gaze and emotional expression (Puce et al., 1998; Narumoto et al., 2001; Pelphrey et al., 2004; Winston et al., 2004; Calder et al., 2007; Engell and Haxby, 2007; Furl et al., 2007).

Beyond specifying the functional properties of each individual node in a network, it may be essential to understand their connections in order to fully characterize face processing in the brain (Wiggett and Downing, 2008; Thomas et al., 2009). One study demonstrated that connectivity between the FG and an extended network of brain regions during face processing is modulated by the nature of the face (Fairhall and Ishai, 2007): in particular, the FG became more coupled with the amygdala during processing of 
emotional faces, and more coupled with orbitofrontal cortex during processing of famous faces. Our study - exploring connectivity between the FG and pSTS - helps inform an ongoing debate about the independence/interactivity of these regions (Allison et al., 1999; Duchaine et al., 2003; Andrews and Ewbank, 2004; Calder and Young, 2005; Fairhall and Ishai, 2007; Vuilleumier and Pourtois, 2007; Fox et al., 2008). Because both the FG and pSTS are responsive to visual face stimuli (Puce et al., 1995, 1996; Kanwisher et al., 1997) and these stimulus-locked responses would be correlated, it is difficult to assess functional connectivity during face processing tasks. Our approach of examining functional connectivity at rest, without external face stimuli, is therefore well-suited to establishing the existence of intrinsic FG-pSTS connectivity (see also Zhang et al., 2009). The robust and selective resting connectivity we observed between the FG and pSTS may help explain the sparseness of evidence for a double dissociation between identity and emotion processing in the brain (Calder and Young, 2005).

\section{RESTING NETWORKS}

The brain can be divided into several networks of brain regions that exhibit correlations in spontaneous activity during rest (Fox and Raichle, 2007). Networks have been identified for a variety of brain systems, including motor cortex (Biswal et al., 1995), visual cortex (Lowe et al., 1998), and auditory cortex (Cordes et al., 2000), and cognitive processes, including attention (Fox et al., 2006a), executive control (Seeley et al., 2007), language (Hampson et al., 2002), and memory (Vincent et al., 2006), as well the "default network" of brain regions activated during rest (Greicius et al., 2003). Brain regions within a given network tend to have related functions, and are activated in unison by relevant tasks. In this context, small-scale category-specific networks may exist within a more global visual network. Our study suggests that partial functional connectivity analyses - where variances from other functional control seeds are removed - may prove especially useful for identifying these more specific networks.

This change may in part explain why we observed robust FG-pSTS resting functional connectivity in contrast to Fairhall and Ishai (2007), who report weak effective connectivity between these two regions using dynamic causal modeling (DCM) during tasks. This apparent discrepancy may also result from several other features of their study: First, they did not obtain strong evidence at the group level that a feedforward model of face processing with no direct FG-pSTS interaction provided a better fit than a model in which the FG causally influences the pSTS (supplemental nonparametric analyses were required to support this claim). Second, such model comparisons do not rule out existence of baseline FG-pSTS interactions, but rather suggest that accounting for them leads to a worse overall model fit. Third, this study is susceptible to the limitations of DCM, including that the validity of conclusions from their study depends on the tasks and stimuli that they used (see Friston et al., 2003). For example, interactions between the FG and pSTS may have been more apparent if dynamic stimuli had been used, given the role of the pSTS in processing biological motion and its sensitivity to such stimuli (Pelphrey et al., 2004; Said et al., 2010). Fourth, their study addressed a different question - how connectivity changes across stimulus conditions (see also Rotshtein et al., 2007; Nummenmaa et al., 2010) - while we assessed the existence of stable intrinsic FG-pSTS connectivity that persists at rest. Indeed, such intrinsic connectivity may be stationary across rest and task states, with task-related activity superimposed (Fox et al., 2006b; Fox and Raichle, 2007; Buckner et al., 2009).

Consistent with this idea, significant FG-pSTS resting connectivity was also observed in another recent study (Zhang et al., 2009). Their findings are consistent with ours and provide converging support for the existence of FG-pSTS resting connectivity. However, there were some limitations of their approach for characterizing the specificity and functional significance of this relationship. For example, to assess the specificity of right FG connectivity they used a control region in contralateral left ventral temporal cortex, and thus connectivity with the FG may reflect hemisphere-specific variance in addition to face-specific variance (e.g., hemispheric differences in global vs. local processing; Fink et al., 1996). Perhaps most importantly, they relied exclusively on exploratory whole-brain contrasts. This approach makes it hard to assess whether the pSTS region exhibiting resting connectivity with the FG is related to the pSTS activated by face stimuli. In contrast, we reported several analyses that help link resting FG connectivity to task activation by face stimuli, including: (1) the spatial overlap between pSTS clusters from the voxelwise FG connectivity map and the localizer task, (2) resting connectivity between FG and pSTS ROIs defined from the localizer, and (3) correlations in the distributed patterns of face activation from the localizer and resting connectivity with the FG in the pSTS. These analyses provide novel evidence of facespecific resting connectivity between the FG and pSTS.

While there have been previous attempts to link resting functional connectivity with task-related processing, these efforts have focused primarily on the impact of recent tasks on resting connectivity (Peltier et al., 2005; Waites et al., 2005; Stevens et al., 2010), or on the ability of resting connectivity to predict behavior (Hampson et al., 2006; Fox et al., 2007; cf. Turk-Browne et al., 2006; Leber et al., 2008). The pattern analysis technique introduced in Experiment 2 may provide a new method for assessing the functional significance of intrinsic interactions. This approach could be applied broadly to help constrain relationships between nodes in established networks (e.g., the frontoparietal attention network), and also help to discover networks in the first place. For example, after finding a region activated by a task, one could search for seeds that produce a pattern of resting connectivity in that region predictive of the pattern of task activation. Interactions with such seeds may be important for the function of the initial region.

\section{FUTURE DIRECTIONS}

Further work will be needed to fully characterize the nature of the relationship between the FG and pSTS. Resting functional connectivity between the FG and pSTS may signify that these two regions are directly interactive. Alternatively, interactions between the FG and pSTS may be mediated by other regions, such as the inferior occipital gyrus or lateral occipital complex. Causal interpretations such as these are inherently underdetermined by correlations in the BOLD response. However, a recent study using combined microstimulation and fMRI demonstrated that electrical stimulation of a face-selective patch in primate inferior temporal cortex resulted in selective BOLD responses in other non-contiguous face patches in the temporal lobe (Moeller et al., 2008). While the 
precise homologies between these patches and human FG/pSTS are unknown, this study nevertheless supports the existence of direct connections between face regions in the temporal lobe. Such connections may be essential for face recognition: individuals with congenital prosopagnosia have disrupted structural connectivity in white matter tracts that run adjacent to the FG into typically face-selective regions of the anterior temporal and frontal lobes (Thomas et al., 2009), accompanied by reduced functional connectivity between the FG and those regions (M. Behrmann, personal communication).

In addition, it is unclear how face perception modulates functional connectivity between the FG and pSTS. If, for example, FG-pSTS interactions help integrate facial identity and expression information, connectivity may change when new individuals or expressions appear in the environment. Such hypotheses may prove difficult to test with resting data, and thus ongoing studies are exploring the dynamics of FG-pSTS functional connectivity during face processing. Finally, while we observed reliable resting connectivity between the FG and pSTS, these regions may differ in terms of stability of their connectivity with other regions. For example, because of its strong selectivity for faces, the FG may exhibit stable connectivity with the same set of face processing regions during both rest and tasks (Zhang et al., 2009), even though the relative strength of these connections may be influenced by stimulus features (Fairhall and Ishai, 2007). In contrast, because the pSTS has been associated with several distinct cognitive processes in addition to face perception - such as biological motion, theory of mind, and multisensory integration - its connectivity with other brain regions may be less stable and depend largely on task demands (Hein and Knight, 2008). Future studies could use our approach to identify the components

\section{REFERENCES}

Aguirre, G. K., Zarahn, E., and D'Esposito, M. (1998). An area within human ventral cortex sensitive to "building" stimuli: evidence and implications. Neuron 21, 373-383.

Allison, T., McCarthy, G., Nobre, A., Puce, A., and Belger, A. (1994). Human extrastriate visual cortex and the perception of faces, words, numbers, and colors. Cereb. Cortex 4, 544-554.

Allison, T., Puce, A., and McCarthy, G. (2000). Social perception from visual cues: Role of the pSTS region. Trends Cogn. Sci. 4, 267-278.

Allison, T., Puce, A., Spencer, D. D., and McCarthy, G. (1999). Electrophysiological studies of human face perception. I: Potentials generated in occipitotemporal cortex by face and non-face stimuli. Cereb. Cortex 9, 415-430.

Andrews, T. J., and Ewbank, M. P. (2004). Distinct representations for facial identity and changeable aspects of faces in the human temporal lobe. NeuroImage 23, 905-913.

Barton, J. J.S., Press, D.Z., Keenan, J.P., and O'Connor, M. (2002). Lesions of the fusiform face area impair perception

of pSTS connectivity. For example, if resting data were collected along with tasks associated with the full range of pSTS functions, connectivity associated with one function (e.g., theory of mind) could be characterized by partialing out the variance from control regions activated by the other functions (e.g., FG for face processing, MT for biological motion, and auditory cortex for multisensory integration). Nevertheless, our initial evidence suggests that the FG may have a special relationship with the pSTS, at least with respect to other category-specific regions of ventral visual cortex.

\section{CONCLUSIONS}

Cognitive neuroscience has recently undergone a revolution with the discovery that the brain can be parcellated into coherent and dynamic functional networks. Similar to our increasingly precise understanding of the functions of individual brain regions, the current results provide initial evidence that the division of the brain into gross sensory, motor, and default networks may underestimate the number and specificity of resting networks. Accordingly, even specific types of information processing - such as face processing - may be reflected in fMRI not only as activation changes in single brain regions, but also in the degree to which these changes are synchronous across regions.

\section{ACKNOWLEDGMENTS}

Supported by NIH R01 MH05286 and P01 NS41328 (Gregory McCarthy) and NSERC PGS-D (Nicholas B. Turk-Browne). The authors would like to thank Marvin Chun, Benjamin Deen, Julie Golomb, Christian Luhmann, and Marcia Johnson for helpful conversations, and James Morris and Michele Diaz for assistance with data collection.

to theory of mind. Neuron 58, 306-324.

Cordes, D., Haughton, V. M., Arfanakis, K., Carew, J. D., Turski, P. A., Moritz, C. H., Quigley, M.A., and Meyerand, M. E. (2001). Frequencies contributing to functional connectivity in the cerebral cortex in "restingstate" data. Am. J. Neuroradiol. 22, 1326-1333.

Cordes, D., Haughton, V. M., Arfanakis, K., Wendt, G. J., Turski, P. A., Moritz, C. H., Quigley, M. A., and Meyerand, M. E. (2000). Mapping functionally related regions of brain with functional connectivity MR imaging. Am. J. Neuroradiol. 21, 1636-1644.

Cox, D., Meyers, E., and Sinha, P. (2004). Contextually evoked object-specific responses in human visual cortex. Science 304, 115-117.

Duchaine, B.C., Parker,H., and Nakayama, K. (2003). Normal recognition of emotion in a prosopagnosic. Perception 32, 827-838.

Engell, A. D., and Haxby, J. V. (2007). Facial expression and gaze-direction in human superior temporal sulcus. Neuropsychologia 45, 3234-3241.

Epstein, R., and Kanwisher, N. (1998). A cortical representation of the local visual environment. Nature 392, 598-601.

Fairhall, S.L., and Ishai,A. (2007). Effective connectivity within the distributed cortical network for face perception. Cereb. Cortex 17, 2400-2406.

Fink, G. R., Halligan, P. W., Marshall, J. C., Frith, C. D., Frackowiak, R. S., and Dolan, R. J. (1996). Where in the brain does visual attention select the forest and the trees? Nature 382, 626-628.

Fox, C. J., Oruç, I., and Barton, J. J. (2008). It doesn't matter how you feel. The facial identity aftereffect is invariant to changes in facial expression. J. Vis. 8, 1-13.

Fox, M. D., Corbetta, M., Snyder, A. Z., Vincent, J. L., and Raichle, M. E. (2006a). Spontaneous neuronal activity distinguishes human dorsal and ventral attention systems. Proc. Natl. Acad. Sci. U.S.A. 103, 10046-10051.

Fox, M. D., Snyder, A. Z., Zacks, J. M., and Raichle, M.E. (2006b). Coherent spontaneous activity accounts for trial-totrial variability in human evoked brain responses. Nat. Neurosci. 9, 23-25.

Fox, M. D., and Raichle, M. E. (2007). Spontaneous fluctuations in brain activity observed with functional 
magnetic resonance imaging. Nat. Rev. Neurosci. 8, 700-711.

Fox, M. D., Snyder, A. Z., Vincent, J. L., Corbetta, M., Van Essen, D. C., and Raichle, M. E. (2005). The human brain is intrinsically organized into dynamic, anticorrelated functional networks. Proc. Natl. Acad. Sci. U.S.A. 102, 9673-9678.

Fox, M.D., Snyder,A.Z., Vincent, J. L., and Raichle, M. E. (2007). Intrinsic fluctuations within cortical systems account for intertrial variability in human behavior. Neuron 56, 171-184.

Friston, K. J., Harrison, L., and Penny, W. (2003). Dynamic causal modelling. NeuroImage 19, 1273-1302.

Furl, N., van Rijsbergen, N. J., Treves, A., Friston, K. J., and Dolan, R. J. (2007). Experience-dependent coding of facial expression in superior temporal sulcus. Proc. Natl. Acad. Sci. U.S.A. 104, 13485-13489.

Ganel, T., Valyear, K. F., GoshenGottstein, Y., and Goodale, M. A. (2005). The involvement of the 'fusiform face area' in processing facial expression. Neuropsychologia 43, 1645-1654.

Gazzaley, A., Cooney, J. W., McEvoy, K., Knight, R. T., and D'Esposito, M. (2005). Top-down enhancement and suppression of the magnitude and speed of neural activity. J. Cogn. Neurosci. 17, 507-517.

Ghazanfar, A. A., and Schroeder, C. E. (2006). Is neocortex essentially multisensory?. Trends Cogn. Sci. 10, 278-285.

Greicius, M. D., Krasnow, B., Reiss, A. L., and Menon, V. (2003). Functional connectivity in the resting brain: A network analysis of the default mode hypothesis. Proc. Natl. Acad. Sci. U.S.A. 100, 253-258.

Hampson, M., Driesen, N. R., Skudlarski, P., Gore, J. C., and Constable, R. T. (2006). Brain connectivity related to working memory performance. $J$. Neurosci. 26, 13338-13343.

Hampson, M., Peterson, B. S., Skudlarski, P., Gatenby, J.C., and Gore, J. C. (2002). Detection of functional connectivity using temporal correlations in MR images. Hum. Brain Mapp. 15, 247-262.

Hanson, S. J., Matsuka, T., and Haxby, J.V. (2004). Combinatorial codes in ventral temporal lobe for object recognition: Haxby (2001) revisited: is there a 'face' area? NeuroImage 23, 156-166.

Hasselmo, M. E., Rolls, E. T., and Baylis, G. C. (1989). The role of expression and identity in the face-selective responses of neurons in the temporal visual cortex of the monkey. Behav. Brain Res. 32, 203-218.

Haxby, J.V., Hoffman, E. A., and Gobbini, M. I. (2000). The distributed human neural system for face perception. Trends Cogn. Sci. 4, 223-233.

He, B. J., Snyder, A. Z., Vincent, J. L., Epstein, A., Shulman, G. L., and Corbetta, M. (2007). Breakdown of functional connectivity in frontoparietal networks underlies behavioral deficits in spatial neglect. Neuron 53, 905-918.

Hein, G., and Knight, R. T. (2008).Superior temporal sulcus-it's my area: Or is it? J. Cogn. Neurosci. 20, 2125-2136.

Ishai, A. (2008). Let's face it: It's a cortical network. NeuroImage 40, 415-419.

Kanwisher, N., McDermott, J., and Chun, M. M. (1997). The fusiform face area: A module in human extrastriate cortex specialized for face perception. $J$. Neurosci. 17, 4302-4311.

Leber, A. B., Turk-Browne, N. B., and Chun, M.M. (2008). Neural predictors of moment-to-moment fluctuations in cognitive flexibility. Proc. Natl. Acad. Sci. U.S.A. 105, 13592-13597.

Lowe, M. J., Mock, B. J., and Sorenson, J. A. (1998). Functional connectivity in single and multislice echoplanar imaging using resting-state fluctuations. NeuroImage 7, 119-132.

McCarthy, G., Puce, A., Gore, J. C., and Allison, T. (1997). Face-specific processing in the human fusiform gyrus. J. Cogn. Neurosci. 9, 605-610.

Meyers, L. S., Gamst, G., and Guarino, A. J. (2006). Applied Multivariate Research. Thousand Oaks, CA: Sage Publications.

Mitchell, J. P. (2008). Activity in right temporo-parietal junction is not selective for theory-of-mind. Cereb. Cortex 18, 262-271.

Moeller, S., Freiwald, W. A., and Tsao, D. Y. (2008). Patches with links: A unified system for processing faces in the macaque temporal lobe. Science 320 , 1355-1359.

Narumoto, J., Okada, T., Sadato, N., Fukui, K., and Yonekura, Y. (2001).Attention to emotion modulates fMRI activity in human right superior temporal sulcus. Cogn. Brain Res. 12, 225-231.

Nir, Y., Hasson, U., Levy, I., Yeshurun, Y., and Malach, R. (2006). Widespread functional connectivity and fMRI fluctuations in human visual cortex in the absence of visual stimulation. NeuroImage 30, 1313-1324.

Nummenmaa, L., Passamonti, L., Rowe, J. Engell, A. D., and Calder, A. J. (2010). Connectivity analysis reveals a cortical network for eye gaze perception. Cereb. Cortex 20, 1780-1787.

Pelphrey, K.A., Viola, R. J., and McCarthy, G. (2004). Processing of mutual and averted social gaze in the superior temporal sulcus. Psychol. Sci. 15, 598-603.

Peltier, S. J., LaConte, S. M., Niyazov, D. M., Liu, J.Z., Sahgal, V., Yue, G. H., and
Hu, X. P. (2005). Reductions in interhemispheric motor cortex functional connectivity after muscle fatigue. Brain Res. 1057, 10-16.

Perrett, D. I., Smith, P. A. J., Potter, D. D., Mistlin, A. J., Head, A. S., Milner, A. D., and Jeeves, M.A. (1984). Neurones responsive to faces in the temporal cortex: Studies of functional organization, sensitivity to identity and relation to perception. Hum. Neurobiol. 3, 197-208.

Poline, J. B., Worsley, K. J., Evans, A. C. and Friston, K. J. (1997). Combining spatial extent and peak intensity to test for activations in functional imaging. NeuroImage 5, 83-96.

Puce, A., Allison, T., Asgari, M., Gore, J. C., and McCarthy, G. (1996). Differential sensitivity of human visual cortex to faces, letterstrings, and textures: A functional magnetic resonance imaging study. J. Neurosci. 16, 5205-5215.

Puce, A., Allison, T., Bentin, S., Gore, J. C. and McCarthy, G. (1998). Temporal cortex activation in humans viewing eye and mouth movements. J. Neurosci. 18, 2188-2199.

Puce, A., Allison, T., Gore, J. C., and McCarthy, G. (1995). Face-sensitive regions in human extrastriate cortex studied by functional MRI. J. Neurophysiol. 74, 1192-1199.

Puce, A., Allison, T., and McCarthy, G. (1999). Electrophysiological studies of human face perception. III: Effects of top-down processing on face-specific potentials. Cereb. Cortex 9, 445-458.

Rotshtein, P., Henson, R. N., Treves, A., Driver, J., and Dolan, R. J. (2005). Morphing Marilyn into Maggie dissociates physical and identity face representations in the brain. Nat. Neurosci. 8, 107-113.

Rotshtein, P., Vuilleumier, P., Winston, J., Driver, J., and Dolan, R. (2007). Distinct and convergent visual processing of high and low spatial frequency information in faces. Cereb. Cortex 17 , 2713-2724.

Said, C. P., Moore, C. D., Norman, K. A., Haxby, J. V., and Todorov, A. (2010). Graded representations of emotional expressions in the left superior temporal sulcus. Front. Syst. Neurosci. 4: 6 doi:10.3389/fnsys.2010.00006.

Saxe, R. (2006). Uniquely human social cognition. Curr. Opin. Neurobiol. 16, 235-239.

Saxe, R., Xiao, D. K., Kovacs, G., Perrett, D. I., and Kanwisher, N. (2004). A region of right posterior superior temporal sulcus responds to observed intentional actions. Neuropsychologia 42, 1435-1446.

Seeley, W. W., Menon, V., Schatzberg, A. F., Keller, J., Glover, G. H., Kenna, H., Reiss, A. L., and Greicius, M. D. (2007) Dissociable intrinsic connectivity networks for salience processing and executive control. J. Neurosci. 27, 2349-2356.

Shulman, G. L., Astafiev, S. V., Franke, D., Pope, D. L. W., Snyder, A. Z., McAvoy, M. P., and Corbetta, M. (2009). Interaction of stimulus-driven reorienting and expectation in ventral and dorsal frontoparietal and basal ganglia-cortical networks. J. Neurosci. 29, 4392-4407.

Stevens, W. D., Buckner, R. L., and Schacter, D. L. (2010). Correlated lowfrequency BOLD fluctuations in the resting human brain are modulated by recent experience in categorypreferential visual regions. Cereb. Cortex 20, 1997-2006

Tabachnick, B. G., and Fidell, L. S. (2007). Using Multivariate Statistics. Boston, MA: Pearson Education.

Thomas, C., Avidan, G., Humphreys, K., Jung, K. J., Gao, F., and Behrmann, M. (2009). Reduced structural connectivity in ventral visual cortex in congenital prosopagnosia. Nat. Neurosci. 12, 29-31.

Turk-Browne, N. B., Yi, D. J., and Chun, M. M. (2006). Linking implicit and explicit memory: Common encoding factors and shared representations. Neuron 49, 917-927.

Vander Wyk, B. C., Hudac, C. M., Carter, E. J., Sobel, D. M., and Pelphrey, K. A. (2009). Action understanding in the superior temporal sulcus region. Psychol. Sci. 20, 771-777.

Vincent, J. L., Snyder, A. Z., Fox, M. D., Shannon, B. J., Andrews, J. R., Raichle, M. E., and Buckner, R. L. (2006). Coherent spontaneous activity identifies a hippocampal-parietal memory network. J. Neurophysiol. 96, 3517-3531.

Vuilleumier, P., and Pourtois, G. (2007). Distributed and interactive brain mechanisms during emotion face perception: Evidence from functional neuroimaging. Neuropsychologia 45, 174-194.

Vuilleumier, P., Richardson, M. P., Armony, J. L., Driver, J., and Dolan, R. J. (2004). Common and distinct neural responses during direct and incidental processing of multiple facial emotions. Nat. Neurosci. 7, 1271-1278.

Waites, A. B., Stanislavsky, A., Abbott, D. F., and Jackson, G. D. (2005). Effect of prior cognitive state on resting state networks measured with functional connectivity. Hum. Brain Mapp. 24, 59-68.

Wiggett, A. J., and Downing, P. E. (2008). The face network: Overextended?. NeuroImage 40, 420-422.

Winston, J. S., Henson, R. N., FineGoulden, M. R., and Dolan, R. J. (2004). fMRI-Adaptation reveals dissociable neural representations of 
identity and expression in face perception. J. Neurophysiol. 92, 1830-1839.

Woolrich, M. W., Behrens, T. E., Beckmann, C. F., Jenkinson, M., and Smith, S. M. (2004). Multilevel linear modelling for FMRI group analysis using Bayesian inference. NeuroImage 21, 1732-1747.

Yi,D.J.,Woodman,G.F., Widders, D.,Marois, R., and Chun, M.M. (2004). Neural fate of ignored stimuli: dissociable effects of perceptual and working memory load. Nat. Neurosci. 7, 992-996.

Zhang, H., Tian, J., Liu, J., Li, J., and Lee, K. (2009). Intrinsically organized network for face perception during the resting state. Neurosci. Lett. 454, 1-5.

Conflict of Interest Statement: The authors declare that the research was conducted in the absence of any commercial or financial relationships that could be construed as a potential conflict of interest.

Received:26May 2010; paperpending published: 03 July 2010; accepted: 23 August 2010; published online: 24 September 2010.

Citation: Turk-Browne NB, NormanHaignere SV and McCarthy G (2010) Face-specific resting functional connectivity between the fusiform gyrus and posterior superior temporal sulcus Front. Hum. Neurosci. 4:176 doi:10.3389/ fnhum.2010.00176.

Copyright @ 2010 Turk-Browne, NormanHaignere and McCarthy. This is an openaccess article subject to an exclusive license agreement between the authors and the Frontiers Research Foundation, which permits unrestricted use, distribution, and reproduction in any medium, provided the original authors and source are credited. 


\section{APPENDIX}

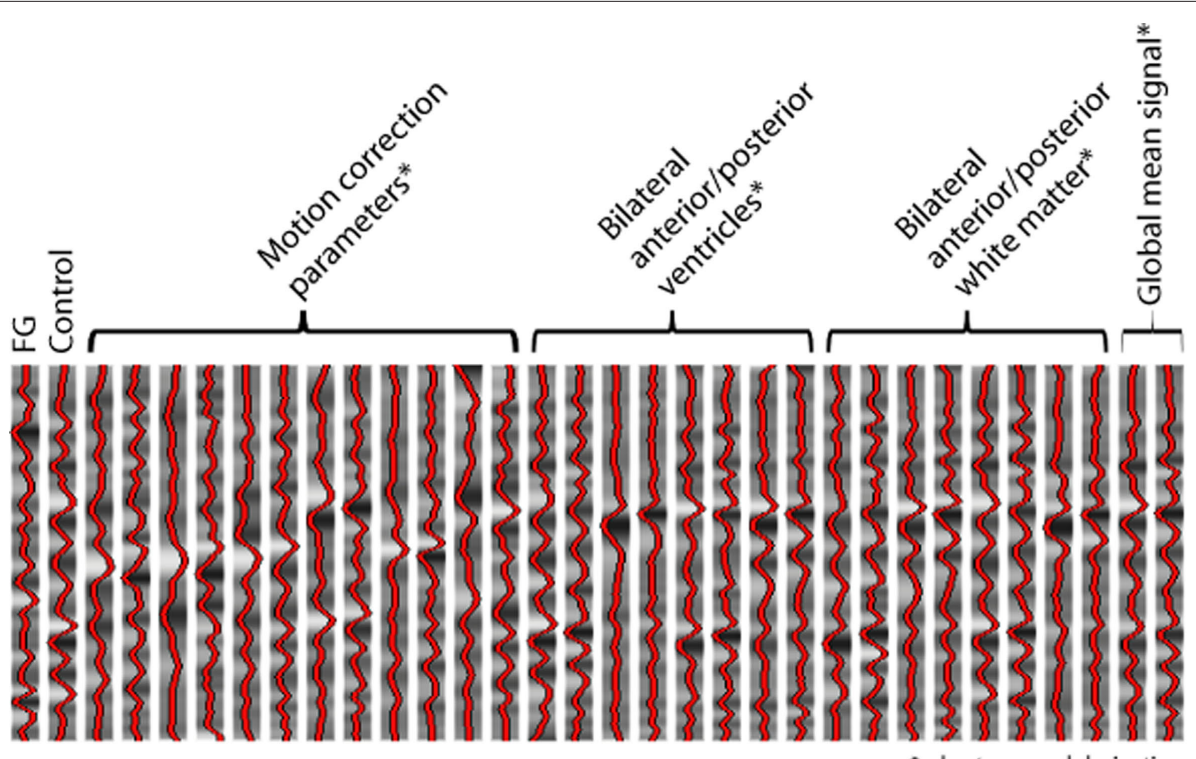

FIGURE A1 | Example resting run model from Experiment 1. Resting timeseries were extracted from the localized FG and control seed regions, and entered into a multiple regression model of the same resting run with several nuisance regressors of no interest.

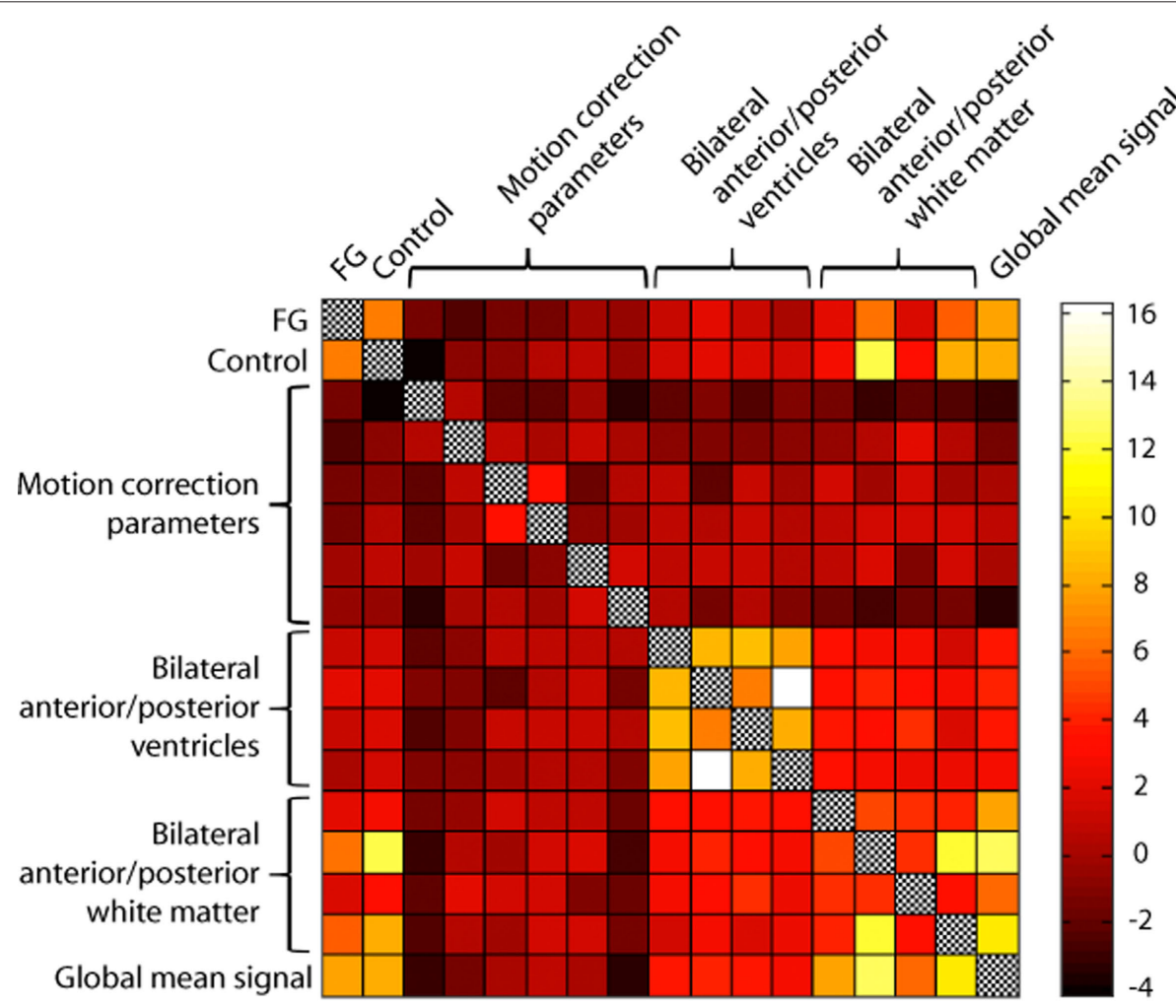

$\mathrm{t}(11)$

FIGURE A2 | Group confusion matrix from Experiment 1. Crosscorrelation of all regressors from resting run models for each subject (concatenating regressors across runs within subject), converted to $z$-scores using Fisher's $r$-to-z transformation, and evaluated across subjects with one-sample $t$-tests. 


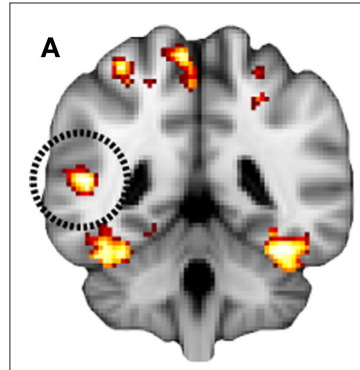

No Kernel

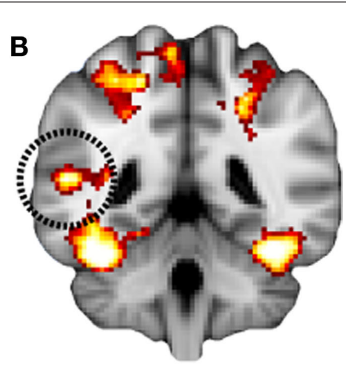

Gaussian Kernel

coronal slice, $y=-44 \mathrm{~mm}(\mathrm{MNI})$

FIGURE A3 | Effect of kernel in Experiment 2. (A) Group analysis of resting correlations with each subject's peak face-selective FG voxel after removing variance from the peak scene-selective PHC voxel. (B) Group analysis of resting correlations when an 8-mm FWHM Gaussian kernel centered on the peak voxel was used to define the seed and control timeseries. 